\title{
CERTAIN NON-INVOLUTORIAL CREMONA TRANSFORMATIONS OF HYPERSPACE
}

BY HARRIET F. MONTAGUE

1. Introduction. The problem here presented was suggested by a paper by Maria Miglio.* A four page synthetic outline given there for $S_{4}$ has been enlarged upon and extended to $S_{r}$ in the present paper.

2. Definition of the Transformation. A non-involutorial Cremona transformation in $S_{r}$ is set up as follows. A $(1,1)$ correspondence is established between the elements of a pencil of primals on a base $o_{1}$ and the points of a rational curve $f_{n}$ of order $n$. A $(1,1)$ correspondence is also established between the elements of a pencil of primals on a base $o_{2}$ and the points of $f_{n}$. Any point $P$ in $S_{r}$ determines with $o_{1}$ an element of the first pencil and associated with this element there is a point $A$ on $f_{n}$. Associated with $A$ there is an element of the second pencil on $o_{2}$. The intersection of this element with the line $A P$ gives $P^{\prime}$, the image of $P$ in the transformation. The inverse transformation proceeds in a similar way starting with the pencil of elements on $o_{2}$.

This transformation is examined as applied to the following cases:

Case A. Pencil of primes on $o_{1}$, pencil of primes on $o_{2}$.

Case B. (a) Pencil of quadric primals on $o_{1}$, pencil of primes on $o_{2}$. primals on $o_{2}$.

(b) Pencil of quadric primals on $o_{1}$, pencil of quadric

Case C. (a) Pencil of cubic primals on $o_{1}$, pencil of primes on $o_{2}$. primals on $o_{2}$.

(b) Pencil of cubic primals on $\boldsymbol{o}_{1}$, pencil of quadric

(c) Pencil of cubic primals on $o_{1}$, pencil of cubic primals on $\boldsymbol{O}_{2}$.

Case D. Pencils of primals of higher order with $f_{n}$ a line $f_{1}$.

* Maria Miglio, Nell' $S_{4}$ una classe di trasformazioni birazionali, Catania Accademia Gioenia Atti, (5), vol. 18 (1932) [Mem. 19], pp. 1-22. 
3. Case A. The transformation is a $T_{n+2}$ and the inverse is of the same order. The locus of invariant points is the locus of intersection of corresponding elements of the two pencils, as is found in all the cases following. The fundamental elements of the transformation are:

(1) The $n+1$ points of $f_{n}$ through which pass the associated primes on $o_{1}$. Their images are the corresponding primes on $\boldsymbol{o}_{2}$.

(2) The $n+1$ intersections of primes on $o_{2}$ which pass through their associated points on $f_{n}$ with the corresponding primes on $o_{1}$. Their images are the $n+1$ primes on $o_{2}$ which pass through their associated points.

(3) The base $o_{1}$. Its image is the projection of a ruled surface of order $n+1$, containing $o_{1}$ once, $o_{2} n$ times, from the common part of $o_{1}$ and $o_{2}$ as vertex.

The residual intersection of two general homaloids, apart from the fundamental elements, is of order $n+2$. In $S_{3}, C_{n+2}$ has $n+1$ points on $o_{1}$, one point on each of the simple fundamental lines, and $n+1$ points on $f_{n}$.

4. Case B. In using a pencil of quadric primals, the point associated with a particular quadric must lie on that quadric. If we have $f_{n}$ a part of the base of the pencil of quadrics, in $S_{3}, n=1,2$, or 3 ; in $S_{r}, r>3, f_{n}$ can be of any order.

(a) A pencil of quadric primals and a pencil of primes gives $T_{2 n+3}$ with the inverse $T_{n+3}^{-1}$.

The image of the base of the pencil of quadrics, a manifold $M_{4}$, is a primal. If $M_{4}$ does not contain $f_{n}, M_{4}$ meets $f_{n}$ in $2 n-1$ points. In $S_{3}$, the image of $C_{4}$ is a surface of order $2 n+5$, containing $o_{2}$ to multiplicity $2 n+1$. If $f_{n}$ is a line $f_{1}, f_{1}$ counts once in the image of $C_{4}$. On the other hand, if $f_{1}$ is a part of the base, the image of the residual $C_{3}$ contains $o_{2}$ once and $f_{1}$ twice.

Whether $f_{n}$ is a part of the base $M_{4}$ or not, the point $A$ lies on its associated quadric. In $S_{3}$ the two generators of the associated quadric which pass through $A$ generate a ruled surface of order $2 n+3$ as $A$ moves along $f_{n}$. This $R_{2 n+3}$ is a principal surface in the transformation.

(b) Two pencils of quadric primals give a $T_{2 n+4}$ with an inverse of the same order. The properties of this transformation may be found in a manner similar to that of (a).

5. Case C. When a pencil of cubic primals is employed, it is 
necessary for a point of $f_{n}$ to be a double point on its associated primal. Then every other point of $f_{n}$ is a simple point of the primal. The transformation is possible only when $f_{n}$ is of order $n=1,2,3$. The discussion is given for $S_{3}$, but the general work may be extended to $S_{r}, r>3$. The author has made the analytic extension, but omits it here for the sake of simplicity. The properties of transformations (b) and (c) may be found in a manner similar to that of (a).

(a) A pencil of cubic primals and a pencil of primes in $S_{3}$. $T_{3 n+4} ; T_{n+4}^{-1}$.

(1) $n=1 . T_{7} ; T_{5}^{-1}$. In $S_{3}$, the line $f_{1}$ is contained twice in the base of the pencil of cubic surfaces, since the surfaces of the pencil touch at every point of $f_{1}$, leaving a residual $C_{7} . C_{7}$ meets $f_{1}$ in 4 points. The image of each point of $C_{7}$ is a conic. The complete image of $C_{7}$ is a surface $j_{10}^{\prime}$ which contains $f_{1}$ counted four times and $o_{2}$ counted three times, that is, $C_{7} \sim j_{10}^{\prime} ; o_{2}{ }^{3} f_{1}{ }^{4}$.

The image of $f_{1}$ is $f_{1}$ itself. There are two points of $f_{1}$ through which pass their associated planes on $o_{2}$. The images of these two points in the inverse transformation are the cubic surfaces associated with these points respectively.

There are two plane cubic curves formed by the intersection of the two planes through their associated points with the corresponding cubic surfaces. These curves are part of the base of the pencil of cubic surfaces. The six lines on any cubic surface which can be drawn from the associated point $A$ generate a ruled surface of order 8 , as $A$ moves along $f_{1}$, and this $R_{8}$ is a principal surface. Every plane through $f_{1}$ intersects $R_{8}$ in a residual $C_{3}$. The curve $C_{3}$ breaks up into the three lines joining in pairs the three points of $C_{7}$ not on $f_{1}$ in the plane.

Two general homaloids of the transformation meet in a variable $C_{5}$. The residual intersection is $C_{7}{ }^{4} f_{1}{ }^{10} \gamma_{3} \delta_{3}$ where $\gamma_{3}$ and $\delta_{3}$ are the two plane cubic curves mentioned above.

(2) $n=2$. $T_{10} ; T_{6}^{-1}$. In $S_{3}$, the conic $f_{2}$ counts twice in the base of the pencil of cubic surfaces, leaving a residual $C_{5}$. The plane of $f_{2}$ meets $C_{5}$ in 5 points, all on $f_{2} . C_{5} \sim j_{10}{ }^{\prime} ; o_{2}{ }^{5}$.

The image of any point $P$ on $f_{2}$ is a conic passing through $P$ and $K$, the intersection of $o_{2}$ and the plane of $f_{2}$, and through the three points of $f_{2}$ through which pass the associated planes on $o_{2}$. The totality of these conics is the image of $f_{2}$. The images, in the inverse transformation, of the three fundamental points of 
$f_{2}$ are the corresponding cubic surfaces. The ruled surface, analogous to $R_{8}$ of the previous case, is, in this case, $R_{11}$.

(3) $n=3 . T_{13} ; T_{7}^{-1}$. In $S_{3}, f_{3}$ counts twice in the base of the pencil of cubic surfaces, leaving a residual $C_{3}$. The curve $C_{3}$ breaks up into three lines which are bisecants of $f_{3} . C_{3} \sim j_{6}^{\prime} ; o_{2}{ }^{3}$. The image of $f_{3}$ is of order 4 , containing $o_{2}$ twice. There are 4 points on $f_{3}$ through which pass associated planes on $o_{2}$. Their images are the corresponding cubic surfaces. The principal ruled surface in this case is $R_{14}$.

(b) A pencil of cubic primals and a pencil of quadric primals give $T_{3 n+5}, n=1,2,3$, with the inverse $T_{2 n+5}^{-1}$.

(c) Two pencils of cubic primals give $T_{3 n+6}, n=1,2,3$, with an inverse of the same order.

6. Case D. If $f_{n}$ is taken as a line $f_{1}$, it can be of any multiplicity on a surface of any order. Consider a pencil of primals of order $s$ on $o_{1}$, and a pencil of primals of order $t$ on $o_{2}$. Take $f_{1}$ such that a primal on $o_{1}$ has an $(s-1)$-fold point at its associated point of $f_{1}$ and a primal on $o_{2}$ has a $(t-1)$-fold point at its associated point. Then a general point of $f_{1}$ is $(s-2)$-fold on every primal of the first pencil, and $(t-2)$-fold on every primal of the second pencil. We obtain a $T_{2 s+t}$ with the inverse $T_{2 t+8}^{-1}$.

The locus of invariant points is, as before, the locus of intersection of corresponding elements of the two pencils. In $S_{3}$, the bases of the two pencils do not intersect. In $S_{r}, r>3$, they do, and any point on the common part is invariant under the transformation.

In $S_{3}$, the residual part of the base is $C_{3 s-2}$ meeting $f_{1}$ in $3 s-5$ points. The image of $C_{3 s-2}$ is of order 6 plus the multiplicity of $f_{1}$. If the elements of the second pencil are planes, $C_{3 s-2} \sim j_{3 s+1}^{\prime}$; $o_{2}{ }^{3} f_{1}{ }^{3 s-5}$. If the elements of the second pencil are surfaces of order $t$, the cone joining $C_{3 s-2}$ to a point $A$ on $f_{1}$ is met by the associated surface of the second pencil in a conical curve of order $(3 s-2) t$, the image of $C_{3 s-2}$ for the particular surface associated with $A$.

In $S_{3}$, if $f_{1}$ is an $(s-1)$-fold line on the first base, the residual part of the base is $C_{2 s-1}$. There is one point of $C_{2 s-1}$ not on $f_{1}$ in every plane through $f_{1}$. The image of $C_{2 s-1}$ is of order 2 plus the multiplicity of $f_{1}$. If the elements of the second pencil are planes, $C_{2 s-1} \sim j_{2 s}^{\prime} ; o_{2}$. If the elements of the second pencil are 
surfaces of order $t$, the image of $C_{2 s-1}$ for a particular surface is a conical curve of order $(2 s-1) t$.

In $S_{r}$, the image of any point on the base $M_{s^{2}}$ or $M_{t^{2}}$ is a conic. The lines joining points of $M$ to a particular point of $f_{1}$ form a conical primal with a point vertex. It is met by the corresponding primal of the second pencil in a manifold $M_{(3 s-2) t}^{r-2}$ or $M_{(2 s-1) t}^{r-2}$ according as there is contact or not.

UNIVERSITY OF BUFFALO

\section{NOTE ON SOME EQUATIONS WITHOUT AFFECT*}

\section{BY SAUNDERS MACLANE}

A numerical equation of degree greater than 4 certainly cannot be solved by radicals if it is "without affect"; that is, if its Galois group is the symmetric group. Hence it is of interest to construct explicitly such equations. A number of such constructions have been developed, $\uparrow$ many of them intrinsically related to certain prime-ideal decompositions. Hence the Newton polygon construction for prime ideals and the related Eisenstein irreducibility criterion are relevant, and can be used systematically to give new proofs for several known constructions (Theorem 2) and for some new equations without affect (Theorems 1 and 2 and generalizations). The advantages lie in the uniform procedure and in the ease of the explicit construction of Thearem 1.

TheOREM 1. Let $p, q$, and $r$ be rational primes and construct

$$
f(x)=x^{n}+a_{1} x^{n-1}+a_{2} x^{n-2}+\cdots+a_{n}, \quad(n \geqq 4),
$$

with rational integral coefficients $a_{i}$ such that: (I) each $a_{i}$ is $d i$ visible by $r$, but $a_{n}$ is not divisible by $r^{2}$; (II) each $a_{i}$ is divisible by $q$, and $a_{n}$ but not $a_{n-1}$ is divisible by $q^{2}$; (III) the highest power $e_{i}$ such that $a_{i}$ is divisible by $p^{e_{i}}$ satisfies

(2) $\quad e_{1} \geqq 1, \quad e_{2}=1, \quad e_{3} \geqq 2, \quad e_{i}-e_{i-1}>e_{i-1}-e_{i-2}$,

* Presented to the Society, April 10, 1936, and subsequently extended.

$\dagger$ Ph. Furtwängler, Ueber Kriterien für irreduzible und für primitive Gleichungen und über die Aufstellung affektfreier Gleichungen, Mathematische Annalen, vol. 85 (1922), pp. 34-40. 